\title{
UPWIND DISCRETIZATION OF THE STEADY NAVIER-STOKES EQUATIONS
}

\author{
BARRY KOREN \\ Centre for Mathematics and Computer Science, PO Box 4079, NL-1009 AB Amsterdam, The Netherlands
}

\section{SUMMARY}

A discretization method is presented for the full, steady, compressible Navier-Stokes equations. The method makes use of quadrilateral finite volumes and consists of an upwind discretization of the convective part and a central discretization of the diffusive part. In the present paper the emphasis lies on the discretization of the convective part. The solution method applied solves the steady equations directly by means of a non-linear relaxation method accelerated by multigrid. The solution method requires the discretization to be continuously differentiable. For two upwind schemes which satisfy this requirement (Osher's and van Leer's scheme), results of a quantitative error analysis are presented. Osher's scheme appears to be increasingly more accurate than van Leer's scheme with increasing Reynolds number. A suitable higher-order accurate discretization of the convection terms is derived. On the basis of this higher-order scheme, to preserve monotonicity, a new limiter is constructed. Numerical results are presented for a subsonic flat plate flow and a supersonic flat plate flow with oblique shock wave-boundary layer interaction. The results obtained agree with the predictions made. Useful properties of the discretization method are that it allows an easy check of false diffusion and that it needs no tuning of parameters.

KEY WORDS Upwind schemes Navier-Stokes equations

\section{INTRODUCTION}

\subsection{Navier-Stokes equations}

The equations considered are the full, steady, 2D, compressible Navier-Stokes equations

$$
\frac{\partial \mathbf{f}(\mathbf{q})}{\partial x}+\frac{\partial \mathbf{g}(\mathbf{q})}{\partial y}-\frac{1}{R e}\left(\frac{\partial \mathbf{r}(\mathbf{q})}{\partial x}+\frac{\partial \mathbf{s}(\mathbf{q})}{\partial y}\right)=\mathbf{0}
$$

with $\mathbf{f}(\mathbf{q})$ and $\mathbf{g}(\mathbf{q})$ the convective flux vectors, Re the Reynolds number, and $\mathbf{r}(\mathbf{q})$ and $\mathbf{s}(\mathbf{q})$ the diffusive flux vectors. As state vector we choose $\mathbf{q}=(\rho, \rho u, \rho v, \rho e)^{T}$, with the total energy $e$ satisfying, (assuming a perfect gas) $e=p /(\rho(\gamma-1))+\frac{1}{2}\left(u^{2}+v^{2}\right)$. The primitive variables used are the density $\rho$, the pressure $p$ and the velocity components $u$ and $v$ in the $x$ - and $y$-direction respectively. The ratio of specific heats $\gamma$ is assumed to be constant. The convective flux vectors are defined by

$$
\mathbf{f}(\mathbf{q})=\left[\begin{array}{l}
\rho u \\
\rho u^{2}+p \\
\rho u v \\
\rho u(e+p / \rho)
\end{array}\right], \quad \mathbf{g}(\mathbf{q})=\left[\begin{array}{l}
\rho v \\
\rho v u \\
\rho v^{2}+p \\
\rho v(e+p / \rho)
\end{array}\right]
$$


and the diffusive flux vectors by

$$
\mathbf{r}(\mathbf{q})=\left[\begin{array}{l}
0 \\
\tau_{x x} \\
\tau_{x y} \\
u \tau_{x x}+v \tau_{x y}+\frac{1}{\gamma-1} \frac{1}{P r} \frac{\partial\left(c^{2}\right)}{\partial x}
\end{array}\right], \quad \mathbf{s}(\mathbf{q})=\left[\begin{array}{l}
0 \\
\tau_{x y} \\
\tau_{y y} \\
v \tau_{y y}+u \tau_{x y}+\frac{1}{\gamma-1} \frac{1}{P r} \frac{\partial\left(c^{2}\right)}{\partial y}
\end{array}\right],
$$

with $\operatorname{Pr}$ the Prandtl number, $c=\sqrt{ }(\gamma p / \rho)$ the speed of sound, and $\tau_{x x}, \tau_{x y}$ and $\tau_{y y}$ the viscous stresses. Assuming the diffusion coefficients to be constant and the Stokes hypothesis to hold, the viscous stresses are given by

$$
\begin{aligned}
\tau_{x x} & =\frac{4}{3} \frac{\partial u}{\partial x}-\frac{2}{3} \frac{\partial v}{\partial y}, \\
\tau_{x y} & =\frac{\partial u}{\partial y}+\frac{\partial v}{\partial x}, \\
\tau_{y y} & =\frac{4}{3} \frac{\partial v}{\partial y}-\frac{2}{3} \frac{\partial u}{\partial x} .
\end{aligned}
$$

In this paper we present a discretization method which allows an accurate (and efficient) computation of (steady) high-Reynolds-number flows up to and including the Euler flow regime. The challenge in developing such a method is to find a discretization of the convective part which is accurate not only for typical Euler flows, but also for typical Navier-Stokes flows such as boundary layer flows. Finding a discretization of the diffusive part which satisfies the same requirements is thought to be easy.

\subsection{Discretization method}

To still allow Euler flow solutions with discontinuities, equations (1) are discretized in integral form. A straightforward and simple discretization of the integral form is obtained by dividing the computational domain $\Omega$ into quadrilateral finite volumes $\Omega_{i, j}$ and by requiring the conservation laws to hold for each finite volume separately:

$$
\oint_{\partial \Omega_{i, j}}\left(\mathbf{f}(\mathbf{q}) n_{x}+\mathbf{g}(\mathbf{q}) n_{y}\right) \mathrm{d} s-\frac{1}{R e} \oint_{\partial \Omega_{i, j}}\left(\mathbf{r}(\mathbf{q}) n_{x}+\mathbf{s}(\mathbf{q}) n_{y}\right) \mathrm{d} s=\mathbf{0}, \quad \forall i, j .
$$

This discretization requires an evaluation of convective and diffusive fluxes at each cell face.

1.2.1. Evaluation of convective fluxes. Based on experience with the Euler equations (see Reference 1 for an overview), for the evaluation of the convective fluxes we prefer an upwind approach following the Godunov principle. ${ }^{2}$ So, along each cell face, the convective flux is assumed to be constant and to be determined by a uniformly constant left and right state only. To the 1D Riemann problem thus obtained an approximate Riemann solver is applied. The choice of the left and right state to be used as input for the approximate Riemann solver determines the accuracy of the convective discretization. First-order accuracy is obtained simply by taking the left and right state equal to that in the corresponding adjacent volume. ${ }^{3,4}$ Higher-order accuracy is obtained by applying low-degree piecewise polynomial state interpolation (MUSCL approach), using two or three adjacent volume states for the left and right state separately. ${ }^{5,6}$ For this flux evaluation we make use of the rotational invariance of the Navier-Stokes equations in order to 
reduce the number of these evaluations per cell face from two to one. A more detailed discussion of the decretization of the convective part is given in Section 2.

1.2.2. Evaluation of diffusive fluxes. For the evaluation of the diffusive fluxes it is necessary to compute $\nabla u, \nabla v$ and $\nabla c^{2}$ at each cell face. To compute for instance $(\nabla u)_{i+1 / 2, j}$, where $i+1 / 2, j$ refers to the cell face separating $\Omega_{i, j}$ and $\Omega_{i+1, j}$, we use the Gauss theorem

$$
\nabla u_{i+1 / 2, j}=\frac{1}{A_{i+1 / 2, j}} \oint_{\partial \Omega_{i+1 / 2, j}} u \text { nd } s,
$$

with $\partial \Omega_{i+1 / 2, j}$ the boundary and $A_{i+1 / 2, j}$ the area of a shifted quadrilateral finite volume $\Omega_{i+1 / 2, j}$ (Figure 1), of which the vertices $\mathrm{z}=(x, y)^{T}$ are defined by

$$
\mathbf{z}_{i, j \pm 1 / 2}=\frac{1}{2}\left(\mathbf{z}_{i-1 / 2, j \pm 1 / 2}+\mathbf{z}_{i+1 / 2, j \pm 1 / 2}\right)
$$

and a similar expression for $\mathbf{z}_{i+1, j \pm 1 / 2}$. The line integral in (6) is approximated by

$$
\begin{aligned}
\oint_{\partial \Omega_{i+1 / 2, j}} u \text { nd } s= & u_{i+1, j}\left(\mathbf{z}_{i+1, j+1 / 2}^{\perp}-\mathbf{z}_{i+1, j-1 / 2}^{\perp}\right)+u_{i+1 / 2, j+1 / 2}\left(\mathbf{z}_{i, j+1 / 2}^{\perp}-\mathbf{z}_{i+1, j+1 / 2}^{\perp}\right) \\
& +u_{i, j}\left(\mathbf{z}_{i, j-1 / 2}^{\perp}-\mathbf{z}_{i, j+1 / 2}^{\perp}\right)+u_{i+1 / 2, j-1 / 2}\left(\mathbf{z}_{i+1, j-1 / 2}^{\perp}-\mathbf{z}_{i, j-1 / 2}^{\perp}\right),
\end{aligned}
$$

with $\mathbf{z}^{\perp} \equiv(y,-x)^{T}$ and with for $u_{i+1 / 2, j \pm 1 / 2}$ the central expression

$$
u_{i+1 / 2, j \pm 1 / 2}=\frac{1}{4}\left(u_{i, j}+u_{i, j \pm 1}+u_{i+1, j}+u_{i+1, j \pm 1}\right) .
$$

Similar expressions are used for the other gradients and other cell faces. For sufficiently smooth grids this central diffusive flux computation is second-order accurate. Notice that by using central expressions such as (9), the directional dependence coming from the cross-derivative terms is neglected. For high-Reynolds-number flows-our interest-no significant gain in solution quality is expected from a biased approach as proposed in Reference 7. Because of the fact that the present diffusive flux evaluation is rather cheap, use of rotational invariance is hardly advantageous and therefore not applied.

\subsection{Solution method}

We give only a brief summary. For a detailed description of the solution method we refer to Reference 8.

For the non-linear system of first-order accurate discretized equations, collective symmetric point Gauss-Seidel relaxation is used. In this relaxation one or more (exact) Newton steps are

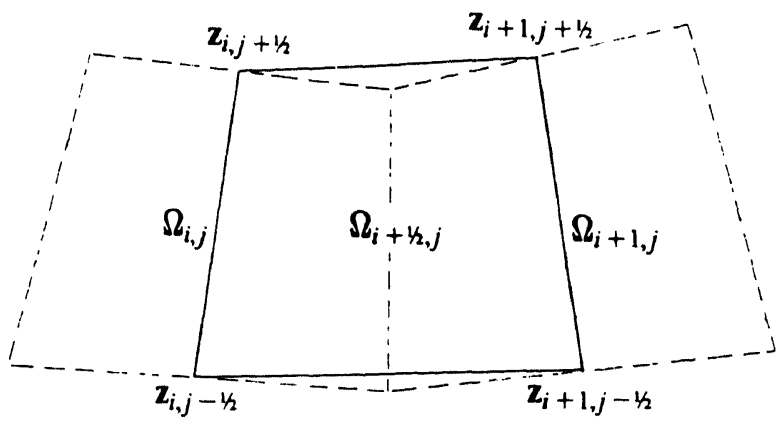

Figure 1. Shifted finite volume $\Omega_{i+1 / 2, j}$ 
used for the collective update of the four state vector components in each finite volume. Nonlinear multigrid is applied as the acceleration technique. The process is started by nested iteration. For the first-order accurate operator this is an efficient process.

For the higher-order accurate operator the same method leads to poor convergence or even divergence. As a remedy we use iterative defect correction as an outer iteration for non-linear multigrid applied-again - to the first-order discretized equations.

The application of the Newton method requires the convective and diffusive fluxes to be continuously differentiable. (The diffusive fluxes as described in the previous section already fulfil this requirement.)

\section{DISCRETIZATION OF CONVECTIVE PART IN MORE DETAIL}

\subsection{Approximate Riemann solver}

As approximate Riemann solver for the Euler equations we prefer Osher's scheme. ${ }^{9}$ Reasons for the preference are (i) its continuous differentiability and (ii) its consistent treatment of boundary conditions. The question arises whether it is still a good choice to use Osher's scheme when, besides discontinuities, also typical Navier-Stokes features such as shear, separation and heat conduction have to be resolved. We therefore reconsider the choice of an approximate Riemann solver for the present application.

Since continuous differentiability is an absolute requirement for the success of our solution method, and since the only known approximate Riemann solvers with this property are Osher's ${ }^{9}$ and van Leer's, ${ }^{10}$ our choice is confined to these two only. So far, van Leer's scheme is more widespread in the field of Navier-Stokes than Osher's. ${ }^{1-13}$ Probably the main reason for this is its greater conceptual and operational simplicity.

In this paper the requirement of accurate modelling of physical diffusion will determine our choice. In Reference 10, van Leer stated already that his flux vector splitter cannot preserve steady contact discontinuities. It diffuses contact discontinuities which, as linear wave phenomena, have no intrinsic steepening mechanism counteracting this diffusion. Since a discrete shear layer may be interpreted as a layer of contact discontinuities, doubt arose about the suitability of van Leer's scheme for Navier-Stokes codes. Recently, this doubt was confirmed in Reference 14, in which a qualitative analysis is presented (supplemented with numerical experiments) for various upwind schemes. There, for the resolution of boundary layer flows, Osher's scheme turns out to be better than van Leer's scheme indeed. To shed some light on the difference in quality, here we present a quantitative error analysis for both Osher's and van Leer's scheme. The analysis is confined to the steady, 2D, isentropic Euler equations for a perfect gas with $\gamma=1$ :

$$
\frac{\partial \mathbf{f}(\mathbf{q})}{\partial x}+\frac{\partial \mathbf{g}(\mathbf{q})}{\partial y}=\mathbf{0}
$$

with

$$
\mathbf{f}(\mathbf{q})=\left[\begin{array}{l}
\rho u \\
\rho\left(u^{2}+c^{2}\right) \\
\rho u v
\end{array}\right], \quad \mathbf{g}(\mathbf{q})=\left[\begin{array}{l}
\rho v \\
\rho v u \\
\rho\left(v^{2}+c^{2}\right)
\end{array}\right],
$$

where $c$ is constant. (The choice of $2 \mathrm{D}$ equations allows us to consider a boundary layer flow in the analysis.) For both upwind schemes we derive the system of modified equations, considering (i) a first-order accurate, finite volume discretization on a square grid (Figure 2) and (ii) a subsonic 


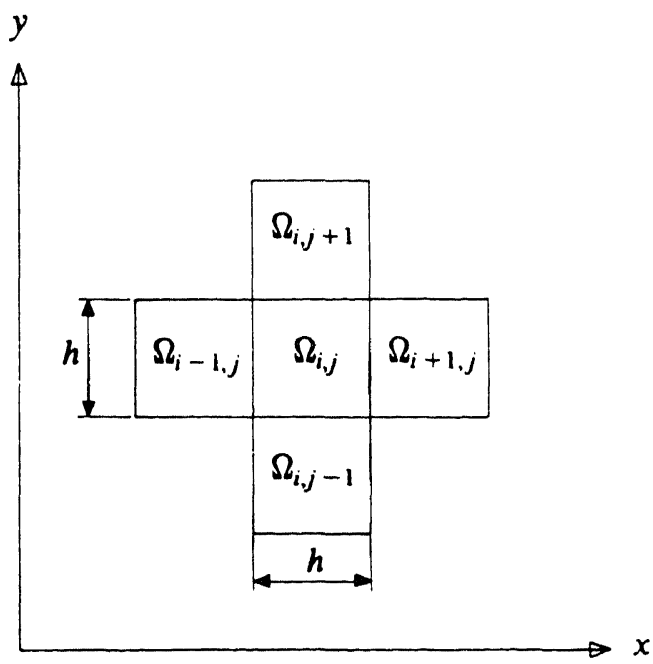

Figure 2. Square model volume $\Omega_{i, j}$ with neighbours

flow with $u$ and $v$ positive and $\rho$ approximately constant. (The discretization is first-order accurate for simplicity and to allow a good display of the differences between the two upwind schemes.)

2.1.1. Osher's scheme. To construct the Osher scheme for the flux vector $\mathbf{f}(\mathbf{q})$ we consider the quasilinear form

$$
\frac{\partial}{\partial t}\left[\begin{array}{l}
\rho \\
u \\
v
\end{array}\right]+\left[\begin{array}{ccc}
u & \rho & 0 \\
c^{2} / \rho & u & 0 \\
0 & 0 & u
\end{array}\right] \frac{\partial}{\partial x}\left[\begin{array}{l}
\rho \\
u \\
v
\end{array}\right]=\mathbf{0}
$$

From this we find directly as the eigenvalues of the Jacobian $\mathrm{d} f(\mathbf{q}) / \mathrm{dq}$

$$
\lambda_{1}=u-c, \quad \lambda_{2}=u, \quad \lambda_{3}=u+c,
$$

and as corresponding eigenvectors

$$
\mathbf{r}_{1}=\left[\begin{array}{c}
\rho \\
-c \\
0
\end{array}\right], \quad \mathbf{r}_{2}=\left[\begin{array}{l}
0 \\
0 \\
1
\end{array}\right], \quad \mathbf{r}_{3}=\left[\begin{array}{l}
\rho \\
c \\
0
\end{array}\right] .
$$

For the Riemann invariants $\psi_{i}^{1}, i=2,3$, satisfying

$$
\rho \frac{\partial \psi_{i}^{1}}{\partial \rho}-c \frac{\partial \psi_{i}^{1}}{\partial u}=0,
$$

we find directly $\psi_{2}^{1}=v$ and by separation of variables $\psi_{3}^{1}=\rho e^{u / c}$. For $\psi_{i}^{2}, i=1,3$, satisfying

$$
\frac{\partial \psi_{i}^{2}}{\partial v}=0
$$


we find directly $\psi_{1}^{2}=\rho$ and $\psi_{3}^{2}=u$. Similarly to $\psi_{i}^{1}$, for $\psi_{i}^{3}, i=1,2$, satisfying

$$
\rho \frac{\partial \psi_{i}^{3}}{\partial \rho}+c \frac{\partial \psi_{i}^{3}}{\partial u}=0
$$

we find $\psi_{1}^{3}=v$ and $\psi_{2}^{3}=\rho e^{-u / c}$. Using then the P-arrangement of subpaths ${ }^{3}$ for $\mathbf{f}(\mathbf{q})$ as given in (11), the Osher scheme can be represented schematically as in Figure 3. Writing Osher's flux function $\mathbf{F}\left(\mathbf{q}_{0}, \mathbf{q}_{1}\right)$ as

$$
\mathbf{F}\left(\mathbf{q}_{0}, \mathbf{q}_{1}\right)=\mathbf{f}\left(\mathbf{q}_{0}\right)+\int_{\mathbf{q}_{0}}^{\mathbf{q}_{1}} \frac{\mathrm{d \mathbf {f } ^ { - }}(\mathbf{q})}{\mathrm{d} \mathbf{q}} \mathrm{d} \mathbf{q},
$$

$\mathrm{df}^{-}(\mathbf{q}) / \mathrm{d} \mathbf{q}$ being the split part of $\mathrm{df}(\mathbf{q}) / \mathrm{d} \mathbf{q}$ which corresponds with the negative eigenvalue $\lambda_{1}$, for the given P-type arrangement of subpaths and for the subsonic flow considered, we find directly

$$
\mathbf{F}\left(\mathbf{q}_{0}, \mathbf{q}_{1}\right)=\mathbf{f}\left(\mathbf{q}_{1 / 3}\right) \text {. }
$$

For $\mathbf{q}_{1 / 3}=\left(\rho_{1 / 3}, u_{1 / 3}, v_{1 / 3}\right)^{T}$, by straightforward arithmetic we find

$$
\mathbf{q}_{1 / 3}=\left[\begin{array}{l}
\sqrt{ }\left(\rho_{0} \rho_{1} e^{\left(u_{0}-u_{1}\right) / c}\right) \\
\frac{1}{2}\left[u_{0}+u_{1}+c \ln \left(\rho_{0} / \rho_{1}\right)\right] \\
v_{0}
\end{array}\right] .
$$

With the simple finite volume grid proposed, and with the neglect of density variation in the error terms, we find then as the system of modified equations for Osher's scheme

$$
\frac{\partial \mathbf{f}(\mathbf{q})}{\partial x}+\frac{\partial \mathbf{g}(\mathbf{q})}{\partial y}-h \frac{\rho}{2 c}\left(\frac{\partial}{\partial x}\left[\begin{array}{c}
\frac{1}{2} \frac{\partial\left(u^{2}\right)}{\partial x} \\
\left(u^{2}+c^{2}\right) \frac{\partial u}{\partial x} \\
u v \frac{\partial u}{\partial x}+u c \frac{\partial v}{\partial x}
\end{array}\right]+\frac{\partial}{\partial y}\left[\begin{array}{c}
\frac{1}{2} \frac{\partial\left(v^{2}\right)}{\partial y} \\
v u \frac{\partial v}{\partial y}+v c \frac{\partial u}{\partial y} \\
\left(v^{2}+c^{2}\right) \frac{\partial v}{\partial y}
\end{array}\right]\right)+O\left(h^{2}\right)=\mathbf{0}
$$

2.1.2. Van Leer's scheme. The flux $\mathbf{f}(\mathbf{q})$ is split into a forward flux $\mathbf{f}^{+}(\mathbf{q})$ and a backward flux $\mathbf{f}^{-}(\mathbf{q})$ such that $\mathrm{df}^{+}(\mathbf{q}) / \mathrm{d} \mathbf{q}$ has all positive eigenvalues and $\mathrm{df}^{-}(\mathbf{q}) / \mathrm{d} \mathbf{q}$ all negative eigenvalues. Additional restrictions imposed on the splitting can be found in Reference 10. For the subsonic

$$
\lambda_{1}=u-c\{\begin{array}{l}
\lambda_{2}=u\left\{\begin{array}{l}
\psi_{1}^{2}=\rho \\
\psi_{3}^{2}=u
\end{array}\right. \\
\psi_{3}^{1}=\rho e^{u / c}
\end{array} \underbrace{}_{0}
$$

Figure 3. Osher path (P-variant) for $\mathbf{f}=\left(\rho u, \rho\left(u^{2}+c^{2}\right), \rho u v\right)^{T}, c=$ constant 
flow considered, van Leer's splitting yields

$$
\mathbf{f}^{+}(\mathbf{q})=\frac{1}{4} \rho \frac{(u+c)^{2}}{c}\left[\begin{array}{l}
1 \\
2 c \\
v
\end{array}\right],
$$

with $\mathbf{f}^{-}(\mathbf{q}) \equiv \mathbf{f}(\mathbf{q})-\mathbf{f}^{+}(\mathbf{q})$. Then, with the square model grid and with the neglect of density variation, we find the following system of modified equations for van Leer's scheme:

$$
\frac{\partial \mathbf{f}(\mathbf{q})}{\partial x}+\frac{\partial \mathbf{g}(\mathbf{q})}{\partial y}-h \frac{\rho}{2 c}\left(\frac{\partial}{\partial x}\left[\begin{array}{l}
\frac{1}{2} \frac{\partial\left(u^{2}\right)}{\partial x} \\
2 c^{2} \frac{\partial u}{\partial x} \\
\frac{1}{2} \frac{\partial\left[\left(u^{2}+c^{2}\right) v\right]}{\partial x}
\end{array}\right]+\frac{\partial}{\partial y}\left[\begin{array}{l}
\frac{1}{2} \frac{\partial\left(v^{2}\right)}{\partial y} \\
\frac{1}{2} \frac{\left.\partial\left(v^{2}+c^{2}\right) u\right]}{\partial y} \\
2 c^{2} \frac{\partial v}{\partial y}
\end{array}\right]\right)+O\left(h^{2}\right)=\mathbf{0}
$$

2.1.3. Error comparison. To compare the error terms in (19) and (21) we consider an incompressible, semi-finite flat plate flow (Figure 4). As flow solution, instead of the analytically intractable Blasius solution, we use Lamb's approximate solution. Lamb's approximation, which shows a better resemblance to the Blasius solution than for instance Pohlhausen's (Figure 5), reads

$$
\left[\begin{array}{l}
\rho \\
u \\
v
\end{array}\right]=\left[\begin{array}{l}
P \\
U \sin \left(\frac{\pi}{2} \frac{\sqrt{ }(\operatorname{Re} / x)}{5} y\right) \\
\frac{y}{2 x} u(x, y)+\frac{5}{\pi} \frac{U}{\sqrt{(R e x)}}\left[\cos \left(\frac{\pi}{2} \frac{\sqrt{ }(\operatorname{Re} / x)}{5} y\right)-1\right]
\end{array}\right],
$$

with $P$ and $U$ constant. Substituting the solution vector (22) into the $O(h)$-error vector of both (19) and (21), considering the boundary layer edge

$$
y=\delta(x) \equiv \frac{5}{\sqrt{(R e / x)}}
$$

at $x=1$, and taking componentwise the ratio of absolute values of both error vectors, using $R e \gg 1$ (our interest), we find

$$
\frac{\text { error (Osher) }}{\text { error (van Leer) }}=\left[\left(1-\frac{2}{\pi}\right) \frac{5}{\sqrt{(R e)}} \frac{U}{c}\right]
$$

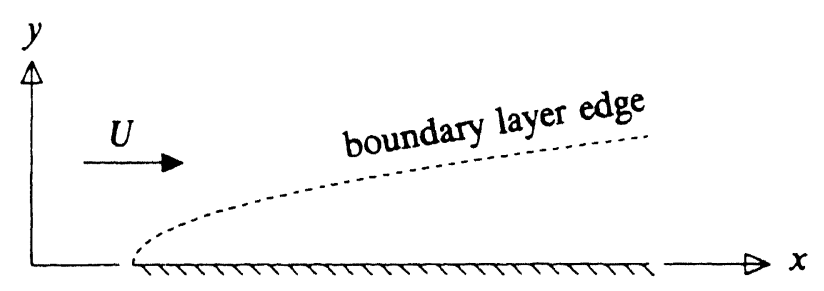

Figure 4. Semi-infinite, incompressible flat plate flow 


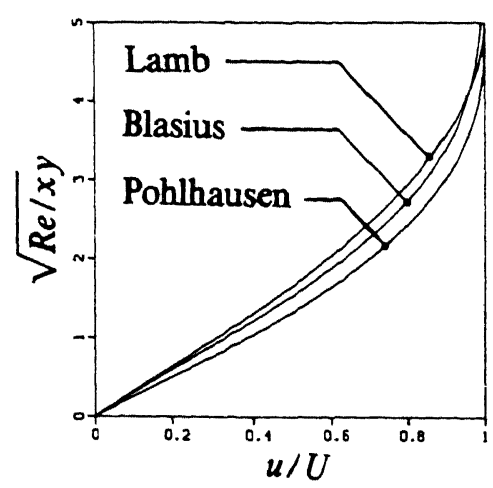

Figure 5. Velocity profiles: exact (Blasius) and approximate (Lamb and Pohlhausen)

From (24) it appears that van Leer's scheme deteriorates compared to Osher's scheme for increasing Re. Assuming (24) to be still reliable for small $R e$, it appears that already for $\operatorname{Re}>[5(1-2 / \pi) U / c]^{2}$, where $U / c<1$, Osher's scheme is to be preferred over van Leer's.

2.1.4. Solid wall boundary condition treatment. To ensure a continuous transition along a solid wall boundary from the Navier-Stokes flow regime to the Euler flow regime, for van Leer's scheme it will be necessary to impose on the convective part only the Euler boundary condition. For a non-permeable solid wall this means that one should only impose a zero normal velocity component on the convective part (in contrast to the diffusive part on which all boundary conditions are to be imposed, i.e. zero normal and tangential velocity components and a temperature condition). By not imposing the no-slip and temperature boundary conditions on the convective part, we avoid that it 'feels' the severe contact discontinuity in the realistic case of a boundary layer flow on a coarse grid and an outer flow with $M$ not small. Such a contact discontinuity will be erroneously spread by van Leer's scheme and cause the solution to be insensitive to $R e$ variation above some finite, rather low value of $R e$.

Osher's scheme can preserve a steady contact discontinuity as long as it is aligned with the grid. Application of (commonly used) body-fitted grids guarantees this alignment along solid walls. Therefore, with a body-fitted grid, Osher's scheme does not need this careful solid wall boundary condition treatment.

\subsection{Higher-order accuracy}

As mentioned in Section 1.2.1, higher-order accuracy is obtained by applying low-degree piecewise polynomial functions through two or three adjacent volume states. The polynomials are given by van Leer's $\kappa$-scheme ${ }^{15}$

$$
\begin{aligned}
& \mathbf{q}_{i+1 / 2, j}^{l}=\mathbf{q}_{i, j}+\frac{1+\kappa}{4}\left(\mathbf{q}_{i+1, j}-\mathbf{q}_{i, j}\right)+\frac{1-\kappa}{4}\left(\mathbf{q}_{i, j}-\mathbf{q}_{i-1, j}\right), \\
& \mathbf{q}_{i+1 / 2, j}^{r}=\mathbf{q}_{i+1, j}+\frac{1+\kappa}{4}\left(\mathbf{q}_{i, j}-\mathbf{q}_{i+1, j}\right)+\frac{1-\kappa}{4}\left(\mathbf{q}_{i+1, j}-\mathbf{q}_{i+2, j}\right),
\end{aligned}
$$

with $\kappa \in[-1,1]$. For $\kappa=-1,0$ and 1 , we have the one-sided, the Fromm and the centred scheme respectively. The aim now is to optimize $\kappa$. For this purpose we consider the scalar model 
equation

$$
\frac{\partial u}{\partial x}+\frac{\partial u}{\partial y}-\varepsilon\left(\frac{\partial^{2} u}{\partial x^{2}}+\frac{\partial u^{2}}{\partial x \partial y}+\frac{\partial^{2} u}{\partial y^{2}}\right)=0 .
$$

On a square grid a finite volume discretization using the $\kappa$-scheme for convection and the central scheme for diffusion yields as the modified equation

$$
\begin{aligned}
\frac{\partial u}{\partial x}+\frac{\partial u}{\partial y}-\varepsilon\left(\frac{\partial^{2} u}{\partial x^{2}}+\frac{\partial^{2} u}{\partial x \partial y}\right. & \left.+\frac{\partial^{2} u}{\partial y^{2}}\right)+h^{2}\left[\frac{\kappa-\frac{1}{3}}{4}\left(\frac{\partial^{3} u}{\partial x^{3}}+\frac{\partial^{3} u}{\partial y^{3}}\right)\right. \\
& \left.-\frac{\varepsilon}{12}\left(\frac{\partial^{4} u}{\partial x^{4}}+2 \frac{\partial^{4} u}{\partial x^{3} \partial y}+2 \frac{\partial^{4} u}{\partial x \partial y^{3}}+\frac{\partial^{4} u}{\partial y^{4}}\right)\right]+O\left(h^{3}\right)=0
\end{aligned}
$$

Assuming the reliability of the underlying Taylor series expansion, from (27) we find as the highest-accuracy value of $\kappa$

$$
\kappa=\frac{1}{3}\left(1+\varepsilon \frac{\frac{\partial^{4} u}{\partial x^{4}}+2 \frac{\partial^{4} u}{\partial x^{3} \partial y}+2 \frac{\partial^{4} u}{\partial x \partial y^{3}}+\frac{\partial^{4} u}{\partial y^{4}}}{\frac{\partial^{3} u}{\partial x^{3}}+\frac{\partial^{3} u}{\partial y^{3}}}\right),
$$

leading to third-order truncation error accuracy. So, even with the second-order accurate diffusive discretization, the complete discretization can be made third-order accurate by choosing this diffusion-dependent $\kappa$. (We notice that with (28), for the evaluation of the higher-order cell face fluxes over the complete computational domain, it is convenient to do this volume by volume in a checkerboard fashion instead of cell face by cell face.) However, since convection-dominated problems (problems with $\varepsilon \ll 1$ ) are our interest, we will simply neglect the above diffusion dependence of $\kappa$ which leads us again to $\kappa=\frac{1}{3}$.

\subsection{Monotonicity}

To preserve monotonicity of the solution we construct a limiter which is consistent with the $\kappa=\frac{1}{3}$-scheme. For this we apply the monotonicity theory of Spekreijse, ${ }^{16}$ an extension of Sweby's theory ${ }^{17}$ which allows more freedom in the limiter construction.

For the limited, higher-order, left and right state vector components we write

$$
\begin{aligned}
& \mathbf{q}_{i+1 / 2, j}^{l(k)}=\mathbf{q}_{i, j}^{(k)}+\frac{1}{2} \psi\left(R_{i, j}^{(k)}\right)\left(\mathbf{q}_{i, j}^{(k)}-\mathbf{q}_{i-1, j}^{(k)}\right), \\
& \mathbf{q}_{i+1 / 2, j}^{r(k)}=\mathbf{q}_{i+1, j}^{(k)}+\frac{1}{2} \psi\left(1 / R_{i+1, j}^{(k)}\right)\left(\mathbf{q}_{i+1, j}^{(k)}-\mathbf{q}_{i+2, j}^{(k)}\right),
\end{aligned}
$$

with $k=1,2,3,4$, with $\psi(R)$ the limiter and with

$$
R_{i, j}^{(k)}=\frac{\mathbf{q}_{i+1, j}^{(k)}-\mathbf{q}_{i, j}^{(k)}}{\mathbf{q}_{i, j}^{(k)}-\mathbf{q}_{i-1, j}^{(k)}}
$$

The limited $\kappa=\frac{1}{3}$-scheme can be written in the one-sided form $(29 \mathrm{a}, \mathrm{b})$ as

$$
\begin{aligned}
& \mathbf{q}_{i+1 / 2, j}^{l(k)}=\mathbf{q}_{i, j}^{(k)}+\frac{1}{2} \xi\left(R_{i, j}^{(k)}\right)\left(\frac{1}{3}+\frac{2}{3} R_{i, j}^{(k)}\right)\left(\mathbf{q}_{i, j}^{(k)}-\mathbf{q}_{i-1, j}^{(k)}\right), \\
& \mathbf{q}_{i+1 / 2, j}^{r(k)}=\mathbf{q}_{i+1, j}^{(k)}+\frac{1}{2} \xi\left(1 / R_{i+1, j}^{(k)}\right)\left(\frac{1}{3}+\frac{2}{3} / R_{i+1, j}^{(k)}\right)\left(\mathbf{q}_{i+1, j}^{(k)}-\mathbf{q}_{i+2, j}^{(k)}\right) .
\end{aligned}
$$

Notice that for $\xi(R)=1$ we have the (non-limited) $\kappa=\frac{1}{3}$-scheme and that $\xi(R)$ defines the limiter $\psi(R)$ by

$$
\psi(R)=\xi(R)\left(\frac{1}{3}+\frac{2}{3} R\right) .
$$


General requirements to be fulfilled by $\xi(R)$ are $\xi(1)=1$ to preserve higher-order accuracy, and $\xi(0)=0$ and boundedness for large $|R|$ to preserve monotonicity. For the latter we require that $\lim _{R \rightarrow \pm} \xi(R)\left(\frac{1}{3}+\frac{2}{3} R\right)=1$. To make the limiter now consistent with the $\kappa=\frac{1}{3}$-scheme we require that $\xi^{\prime}(1)=0$. (This makes the limiter tangent to the $\kappa=\frac{1}{3}$-scheme at $R=1$ in the monotonicity domain.) Imposing these five requirements on the general form

$$
\xi(R)=\frac{\alpha_{1} R^{2}+\alpha_{2} R+\alpha_{3}}{\alpha_{4} R^{2}+\alpha_{5} R+1},
$$

we find with (32)

$$
\psi(R)=\frac{2 R^{2}+R}{2 R^{2}-R+2} .
$$

An illustration of this limiter is given in Figure 6.

\section{NUMERICAL RESULTS}

\subsection{Flow problems}

To evaluate the discretization method, the following flow problems are considered: (i) a subsonic flat plate flow at $M=0.5$ and with $R e$ ranging from $10^{2}$ up to $10^{100}$; (ii) a supersonic flat plate flow with oblique shock wave-boundary layer interaction at $M=2, R e=2.96 \times 10^{5}$. The latter problem stems from Reference 18.

For the subsonic flow problem the Blasius solution is used as a reference. Geometry and boundary conditions for this flow problem are given in Figure 7. For convection the eastern boundary is assumed to be an outflow boundary. For diffusion the northern, southern and eastern boundaries are assumed to be far-field boundaries with zero diffusion. The grids applied are all composed of square finite volumes. As coarsest grid in all multigrid computations we use the $4 \times 2$ grid given in Figure 7 .

For the supersonic flow problem the experimental results from Reference 18 will serve as a reference. Geometry and boundary conditions are given globally in Figure 8. For this problem, in all multigrid computations a $5 \times 2$-grid is applied as the coarsest grid (Figure 8 ). The grid was optimized for convection by introducing a stretching in the flow direction, and in particular by

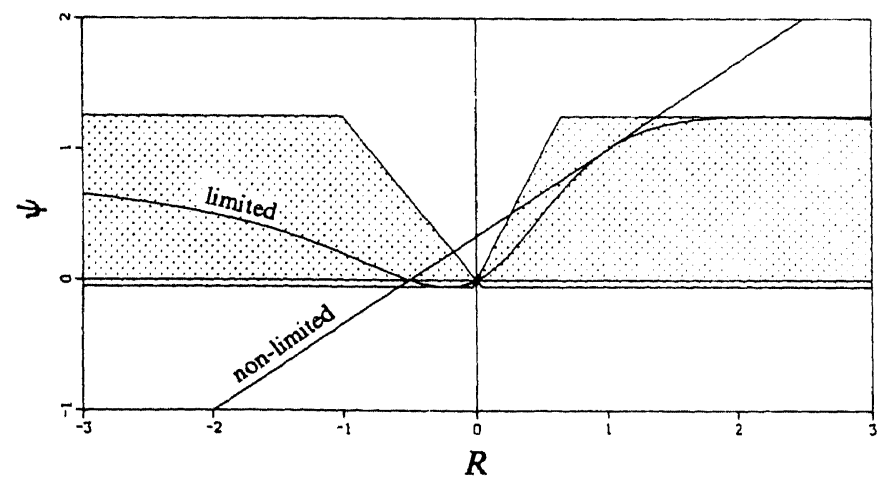

Figure 6. Monotonicity domain with limited and non-limited $\kappa=\frac{1}{3}$-scheme 
conv: $p=1$

diff: zero

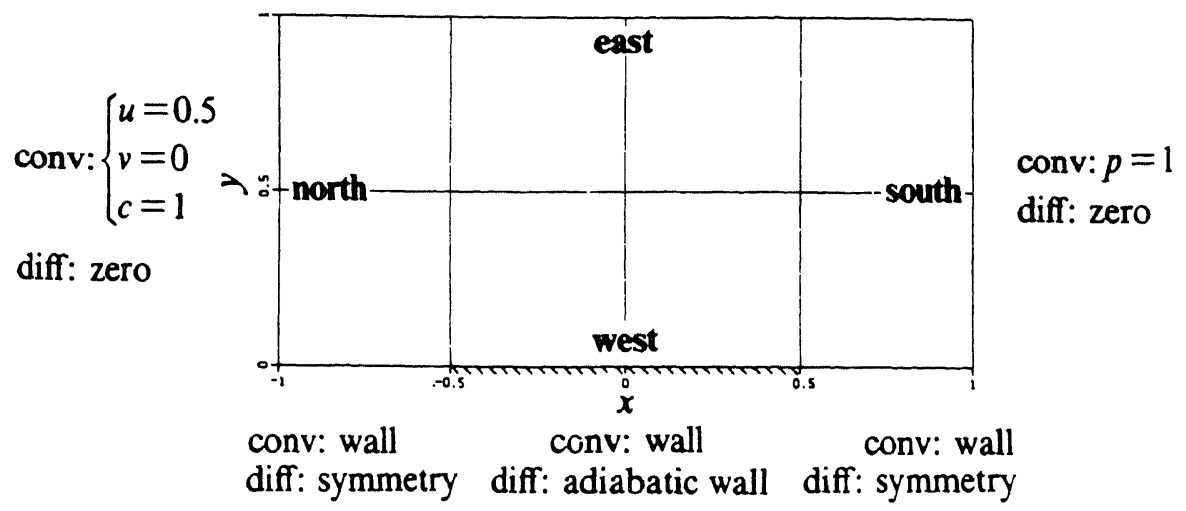

Figure 7. Geometry, boundary conditions and coarsest grid for subsonic flat plate flow (conv: convection, diff: diffusion)
conv: $\begin{cases}u=1, & v=0 \\ c=1 / 2, & p=1\end{cases}$
conv: $\begin{cases}u=U, & v=V \\ c=C, & \rho=R\end{cases}$

diff: zero

diff: zero

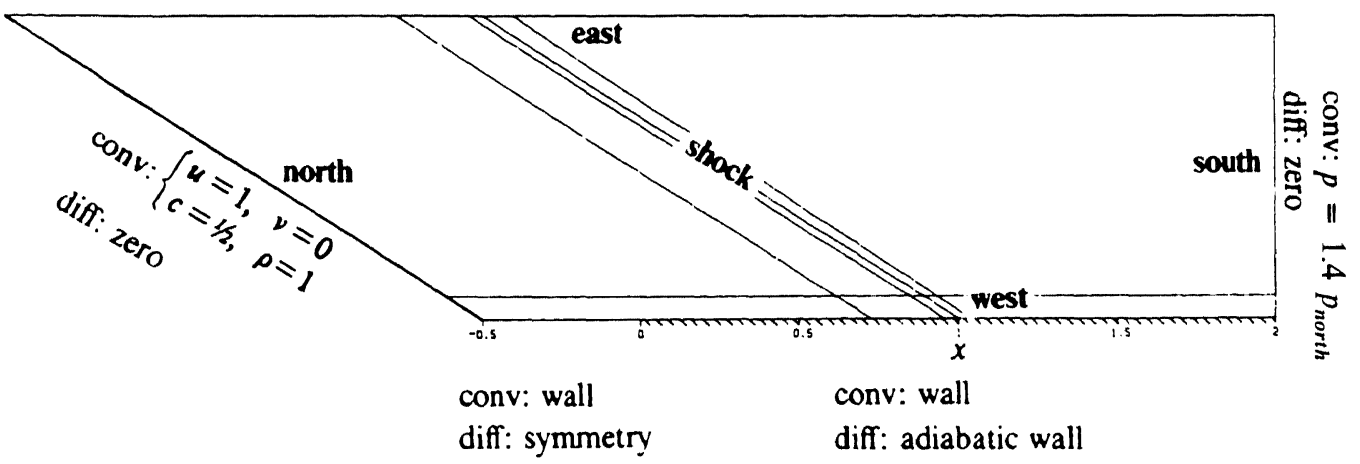

Figure 8. Geometry, boundary conditions and coarsest grid for supersonic flat plate flow (conv: convection, diff: diffusion)

aligning it with the impinging shock wave. A grid adaptation for diffusion was realized by introducing a stretching in the cross-flow direction.

For both flow problems we use $\gamma=1.4$ and $\operatorname{Pr}=0.71$.

\subsection{Osher versus van Leer}

To show at first the benefit of the careful solid wall boundary condition treatment as proposed for van Leer's scheme in Section 2.1.4, we consider the subsonic flat plate flow at $R e=10^{100}$. For both Osher's and van Leer's scheme we compute the flow on a $64 \times 32$ grid $(h=1 / 32$, Figure 9) using the first-order accurate discretization and imposing on the convective part, successively, (i) non-permeability, no slip and no heat transfer, and-carefully-(ii) non-permeability only. As numerical results we show the converged velocity profiles obtained at the middle of the plate $(x=0)$. For the case with all Navier-Stokes boundary conditions imposed it appears that van 


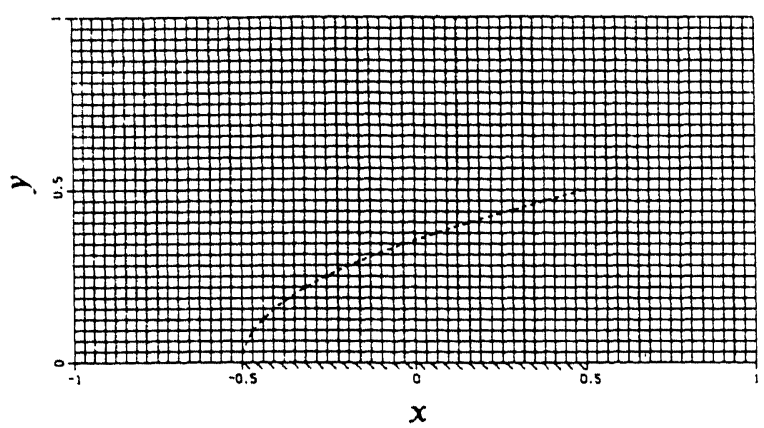

Figure 9. $64 \times 32$ grid for subsonic flat plate flow: $\ldots . .$. , boundary layer edge Blasius solution at $R e=100$

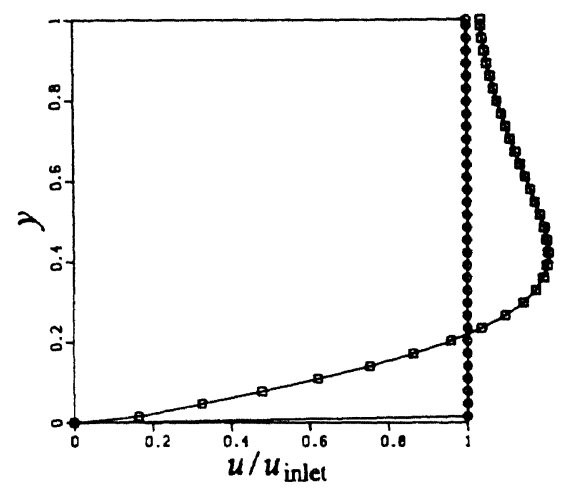

a. $u=v=0, \frac{\partial c}{\partial y}=0$.

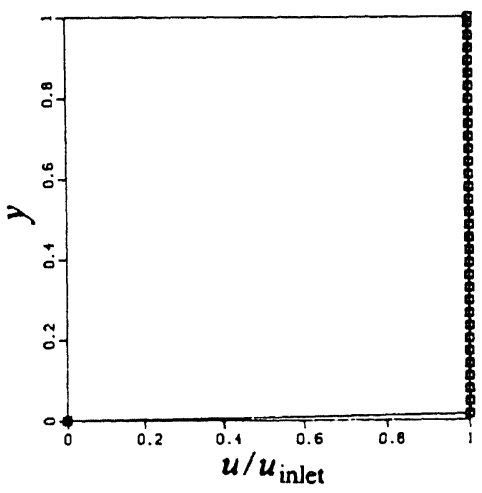

b. $\quad v=0$ only.

Figure 10. Velocity profiles at $x=0$ for subsonic flat plate flow at $R e=10^{100}$ and $h=1 / 32$ for two solid wall boundary condition treatments: $O$, Osher; $\square$, van Leer

Leer's scheme severely thickens the thin layer, whereas Osher's scheme preserves it (Figure 10(a)). With the careful approach, both schemes preserve the layer (Figure 10(b)).

Using the careful boundary condition treatment, for both schemes we perform next an experiment with $h$ and $R e$ variation, using again the first-order accurate discretization. Numerical results obtained-again velocity profiles obtained at $x=0$-are given in Figure 11 . The results show the superiority of Osher's scheme, in particular for high mesh Reynolds numbers. The deterioration of van Leer's scheme with respect to Osher's scheme which occurs in Figure 11(b) for increasing $R e$ is in qualitative agreement with the theoretical results presented in Section 2.1.3.

All further numerical results are obtained with Osher's scheme only.

\subsection{Monotone higher-order accuracy}

To evaluate our monotone higher-order accurate discretization, we consider the supersonic flat plate flow. First we evaluate monotonicity, then higher-order accuracy.

To investigate the monotonicity-preserving properties of our new limiter (34), we compute the Euler flow solution on the $80 \times 32$ grid in Figure 12 using the $\kappa=\frac{1}{3}$-scheme with and without 

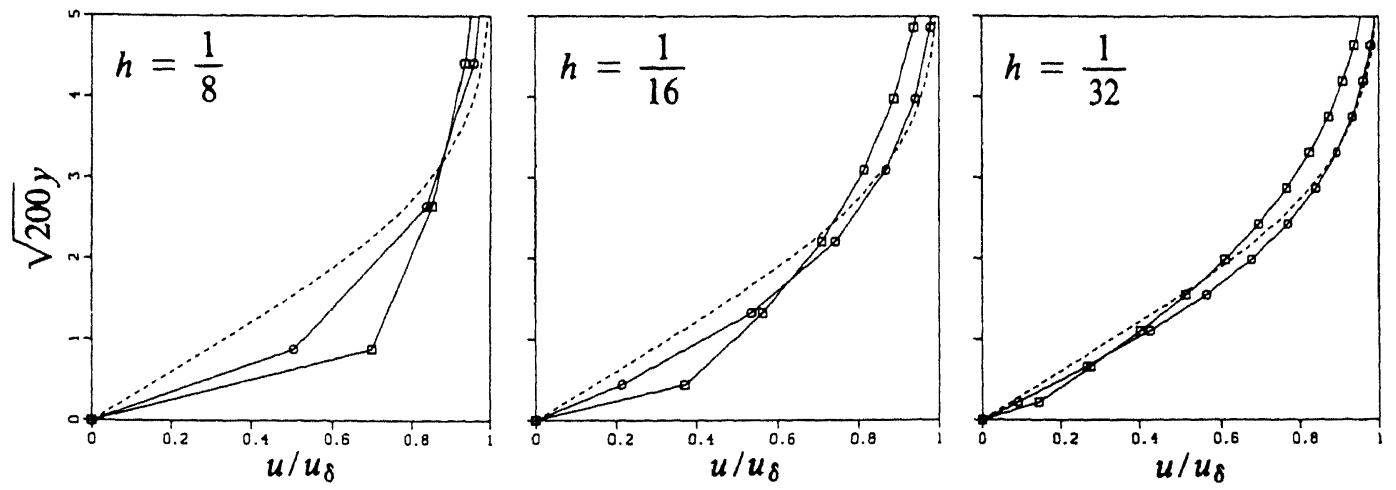

a. $h$-variation at $\operatorname{Re}=100, h=1 / 8,1 / 16,1 / 32$

$$
\text { ( } O \text { : Osher, } \square \text { : Van Leer) }
$$
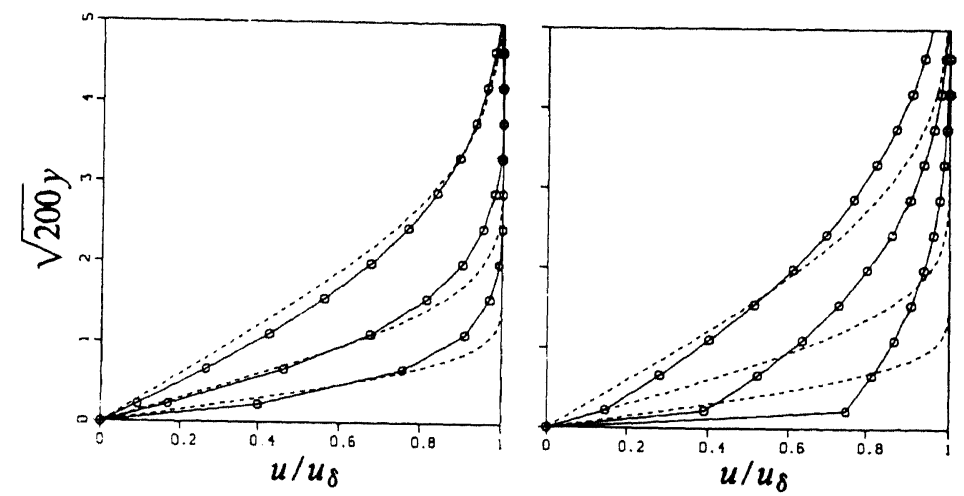

b. $R e$-variation at $h=1 / 32, R e=100,400,1600$

(left: Osher, right: Van Leer)

Figure 11. Velocity profiles at $x=0$ for subsonic flat plate flow: $-\ldots$..., Blasius solution

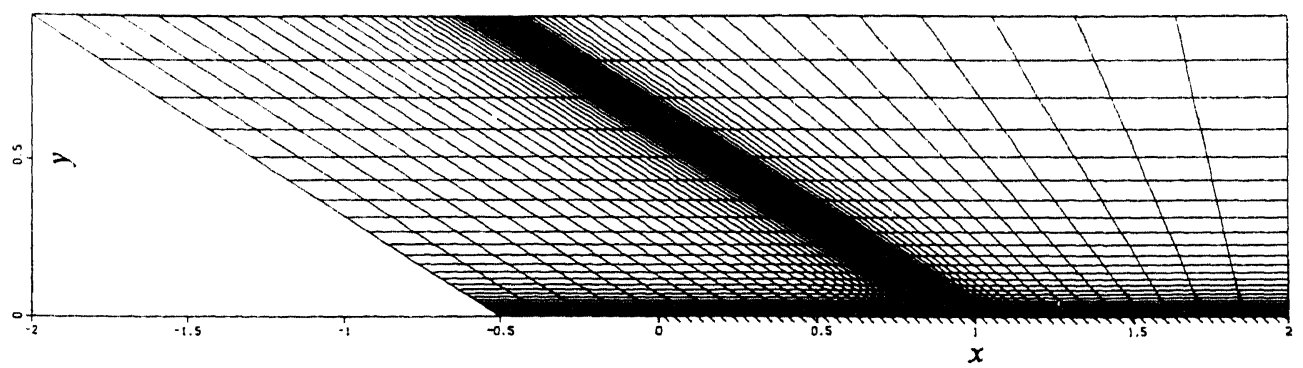

Figure 12. $80 \times 32$ grid for supersonic flat plate flow 


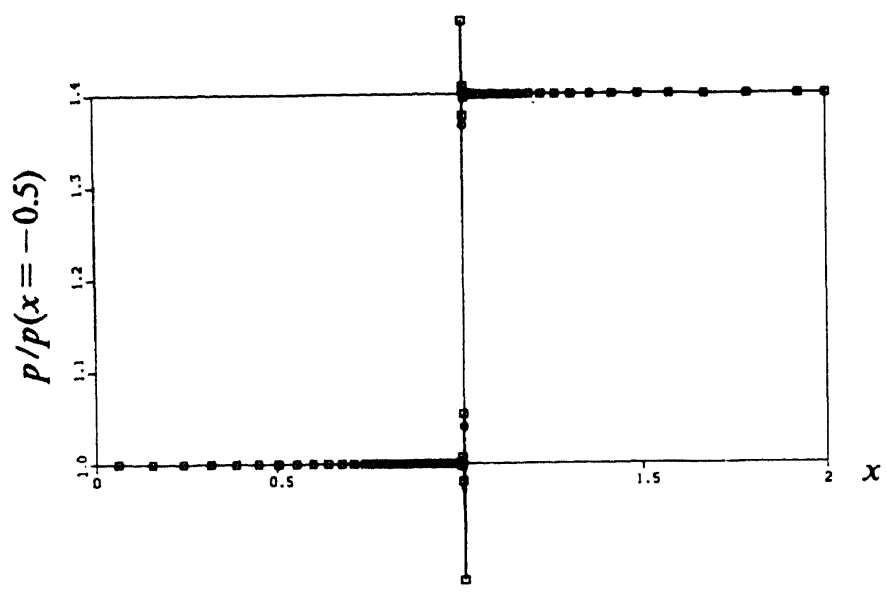

Figure 13. Inviscid surface pressure distributions for supersonic flat plate flow, $80 \times 32$ grid: $\bigcirc$, limited $\kappa=\frac{1}{3}$; $\square$, nonlimited $\kappa=\frac{1}{3}$

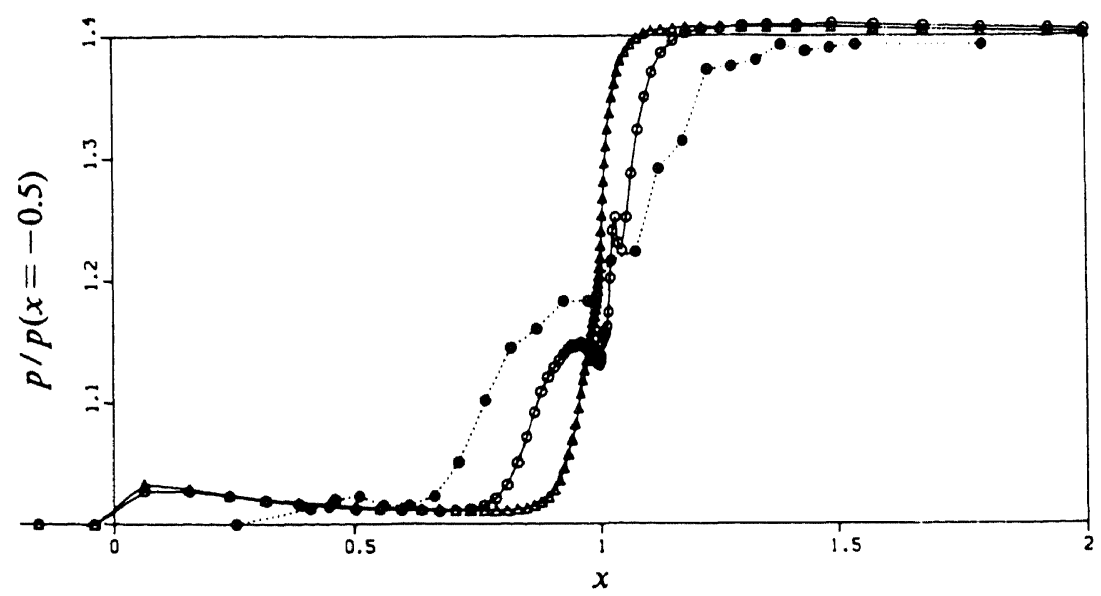

Figure 14. Viscous surface pressure distributions for supersonic flat plate flow, $R e=2.96 \times 10^{5}, 80 \times 32$ grid: $\triangle$, firstorder; $\bigcirc$, limited $\kappa=\frac{1}{3} ; \bullet$, measured

limiter. Numerical results obtained are given in Figure 13. The results clearly show that the limiter does what it is supposed to do: it makes the solution monotone.

To investigate next the accuracy properties of the limited $\kappa=\frac{1}{3}$-discretization, we compute the Navier-Stokes solution on the same grid using the limited $\kappa=\frac{1}{3}$-scheme and the first-order scheme. A comparison is made with the experimental results from Reference 18. Comparing the higher-order surface pressure distribution and the corresponding first-order distribution (Figure 14), a large qualitative difference in the interaction region is clearly visible. The first-order pressure distribution hardly has a plateau in the pressure distribution, which indicates that the corresponding first-order separation bubble is significantly smaller than the higher-order one. A qualitative difference between the higher-order and experimental results (see also Figure 14) seems to be the expansion at the end of the pressure plateau. Though we assume it to be unlikely that in the experiment the expansion lies just in between two neighbouring pressure taps (because 


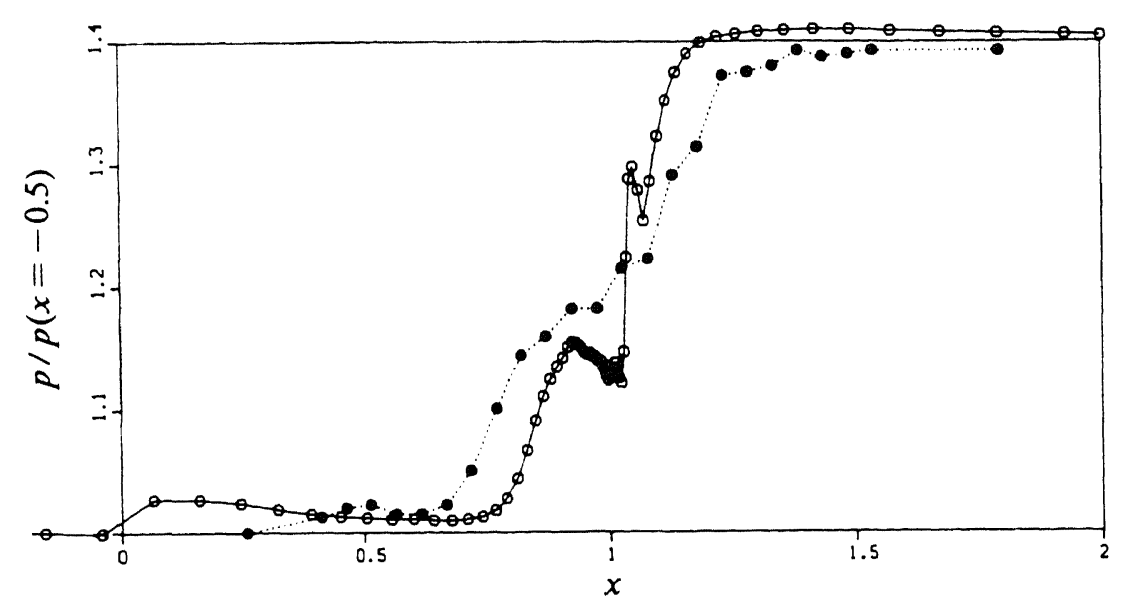

Figure 15. Viscous surface pressure distributions for supersonic flat plate flow, $R e=2.96 \times 10^{5}, 80 \times 32$ grid: $O$, van Albada; $\bullet$, measured

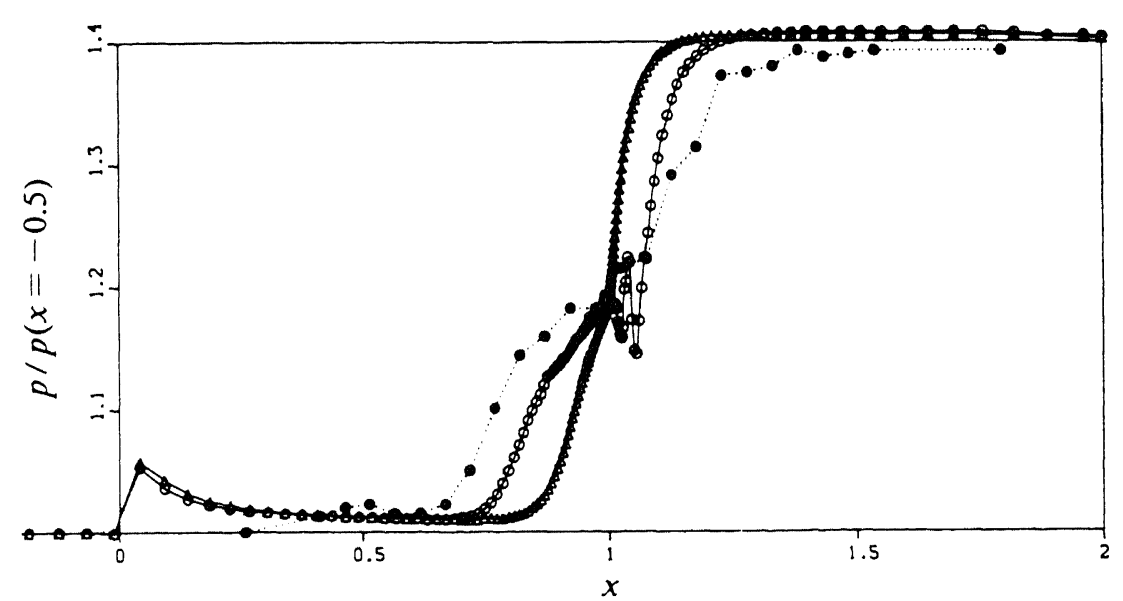

Figure 16. Viscous surface pressure distributions for supersonic flat plate flow, $R e=2.96 \times 10^{5}, 160 \times 64$ grid: $\Delta$, firstorder; $\bigcirc$, limited $\kappa=\frac{1}{3} ; \bullet$, measured

of the absence of an expansion in any other such experiment), we are not (yet) convinced that the expansion is nothing but a numerical artefact. A separation bubble in a supersonic flow has expansion waves emanating above its convex side. As in our higher-order solution, the presence of these waves might well be perceptible on the underlying surface. The wiggles in the higherorder distribution are assumed to be caused by the fact that the limiter cannot be applied near boundaries. To show that they are probably not a deficiency of the new limiter (34), in Figure 15 we give the converged higher-order surface pressure distribution as obtained with the well established van Albada limiter ${ }^{19}$

$$
\psi(R)=\frac{R^{2}+R}{R^{2}+1}
$$

The van Albada distribution appears to be oscillatory as well. 
To investigate whether the quantitative differences existing between the higher-order and measured surface pressure distribution (such as the interaction length) are grid-independent errors of which the cause is not clear, we also compute the limited higher-order solution on a grid twice as fine: the $160 \times 64$ grid corresponding to the $80 \times 32$ grid given in Figure 12. (Uncertain error sources in the wind tunnel experiment might be cross-flow influences, non-observed but non-negligible turbulence, some slight heat transfer through the wall, and so on. For the computation, an error source might for example be the neglect of temperature dependence in the diffusion coefficients.) The converged higher-order surface pressure distribution obtained on the

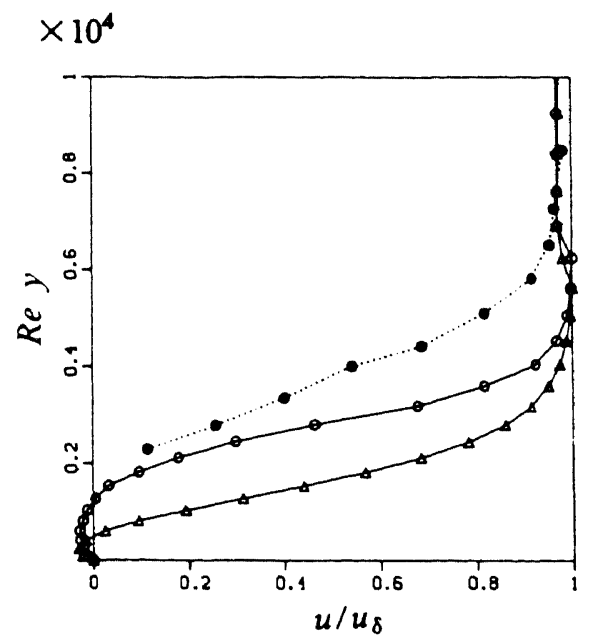

a. In full.

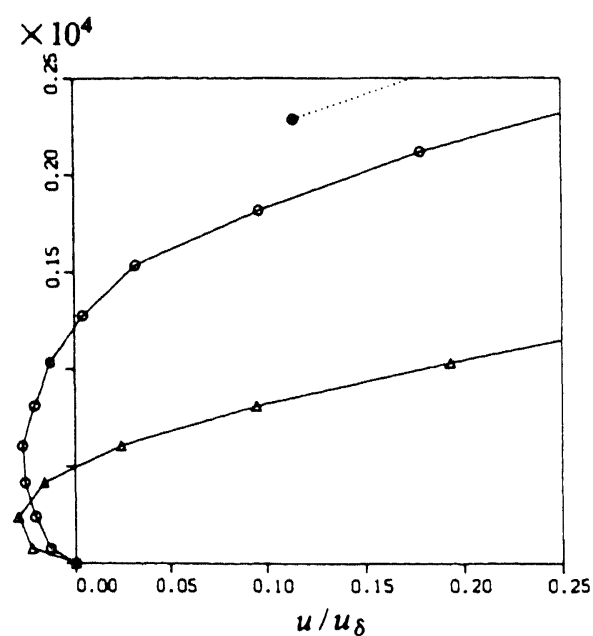

b. In detail.

Figure 17. Velocity profiles at $x=2 \cdot 87 \times 10^{5} / R e$ for supersonic flat plate flow, $R e=2.96 \times 10^{5}, 160 \times 64$ grid: $\triangle$, firstorder; $\bigcirc$, limited $\kappa=\frac{1}{3} ; \bullet$, measured

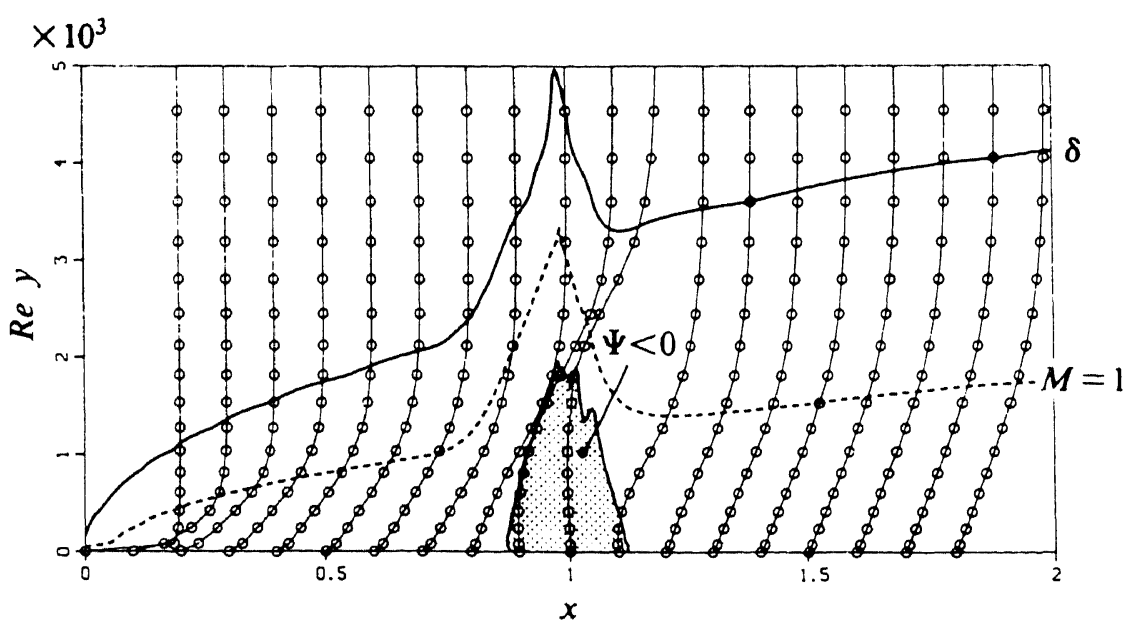

Figure 18. Velocity profiles $(O)$, boundary layer edge $(\delta)$, sonic line $(M=1)$ and separation bubble $(\psi<0)$ for supersonic flat plate flow; limited $\kappa=\frac{1}{3}, R e=2.96 \times 10^{5}, 160 \times 64$ grid 


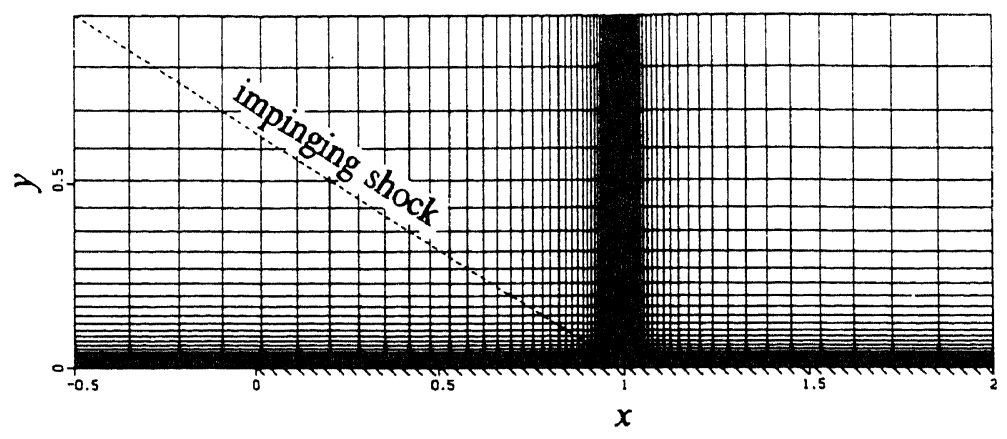

a. Grid

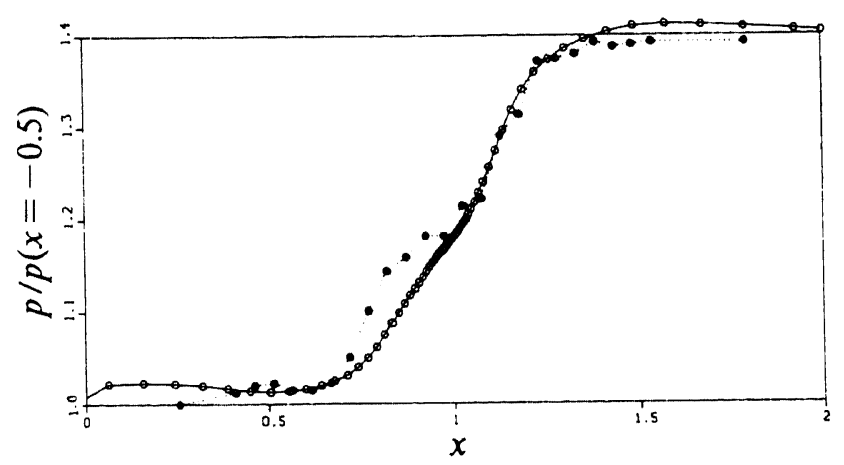

b. Viscous surface pressure distribution

$(\bigcirc:$ limited $\kappa=1 / 3, \bullet:$ measured)

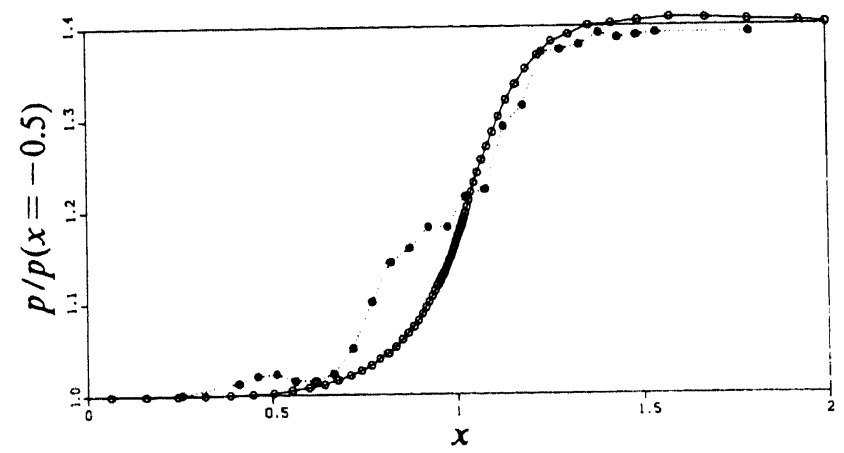

c. Inviscid surface pressure distribution

$$
\text { ( } \bigcirc: \text { limited } \kappa=1 / 3, \bullet: \text { measured) }
$$

Figure 19. Results for supersonic flat plate flow on rectangular $80 \times 32$ grid 
$160 \times 64$ grid is given in Figure 16. Comparison with the measured pressure distribution clearly shows a better resemblance than in the case of the $80 \times 32$ grid (Figure 14), which indicates a probably rather small influence of the uncertain error sources. The converged first-order distribution is also given. Notice again the rather large qualitative difference between the two computed distributions, the first-order one still being without a significant pressure plateau (though also without wiggles). In Figure 17 we also compare velocity profiles at an $x$-location inside the bubble. The smoothness of the higher-order velocity profile seems to justify the previous statement made about the oscillatory surface pressure distribution and the fact that limiters do not work near boundaries. The local maximum in both computed velocity profiles (Figure 17(a)) probably corresponds to the expansion of the supersonic flow over the convex side of the bubble. Further, in Figure 18 we give a more global impression of the higher-order solution.

\subsection{False diffusion}

By presenting for the $80 \times 32$ grid not only the viscous solution obtained with the limited $\kappa=\frac{1}{3}$-scheme (Figure 14) but also the corresponding inviscid solution (Figure 13), insight was given about the amount of false diffusion present in the viscous solution. The fact that the present method can be used for both Navier-Stokes and Euler flows makes this comparison easy. Making the comparison is important. For example, when applying for the supersonic flat plate flow a commonly used rectangular grid, such as the rectangular equivalent of the grid in Figure 12 (Figure 19(a)), a viscous surface pressure distribution is obtained which seems to be very close to the experimental data (Figure 19(b)). However, the corresponding inviscid distribution (Figure 19(c)) indicates that this good resemblance is mainly caused by false numerical diffusion in the discretization of the convective terms and hence is deceptive. In the many numerical results available in the literature for this specific Hakkinen test case we have only seen that rectangular grids (like that in Figure 19(a)) were applied. However, we have not seen any publication in which the corresponding inviscid solution is also shown.

\section{CONCLUSIONS}

With increasing Reynolds number, compared with van Leer's scheme, Osher's scheme appears to lead to an increasingly more accurate resolution of boundary layer flows, in agreement with the theoretical results of Section 2.1.3. Already for rather low Reynolds numbers the difference in accuracy is such that Osher's scheme is to be preferred for engineering purposes. An accidental circumstance is that Osher's scheme needs no special care in the application of solid wall boundary conditions, whereas van Leer's scheme does.

It is important to investigate the reliability of any computed Navier-Stokes solution with respect to the numerical errors in the discretization of the convective part. The present method allows an easy check of false diffusion: the same algorithm can be used for both viscous $(1 / \operatorname{Re}>0)$ and inviscid $(1 / R e=0)$ flow computations.

The discretization method lends itself to efficient solution by multigrid methods and is parameter-free; it needs no tuning.

\section{ACKNOWLEDGEMENT}

This work has been supported by the European Space Agency (ESA) via Avions Marcel Dassault-Bréguet Aviation (AMD-BA). 


\section{REFERENCES}

1. P. W. Hemker and B. Koren, 'Defect correction and nonlinear multigrid for the steady Euler equations', Lecture Series Computational Fluid Dynamics, Von Karman Institute for Fluid Dynamics, Rhode-Saint-Genèse, 1988.

2. S. K. Godunov, 'Finite difference method for numerical computation of discontinuous solutions of the equations of fluid dynamics' (Cornell Aeronautical Lab. Transl. from Russian), Math. Sbornik, 47, 271-306 (1959).

3. P. W. Hemker and S. P. Spekreijse, 'Multiple grid and Osher's scheme for the efficient solution of the steady Euler equations', Appl. Numer. Math., 2, 475-493 (1986).

4. B. Koren, 'Euler flow solutions for transonic shock wave-boundary layer interaction', Int. j. numer. methods fluids, 9, 59-73 (1989)

5. P. W. Hemker, 'Defect correction and higher order schemes for the multi grid solution of the steady Euler equations', Proc. 2nd Eur. Conf. on Multigrid Methods, Cologne, 1985, Lecture Notes in Mathematics, Vol. 1228, Springer, Berlin, 1986, pp. $149-165$.

6. B. Koren, 'Defect correction and multigrid for an efficient and accurate computation of airfoil flows', J. Comput. Phys., 77, 183-206 (1988)

7. S. R. Chakravarthy, K.-Y. Szema, U. C. Goldberg, J. J. Gorski and S. Osher, 'Application of a new class of high accuracy TVD schemes to the Navier-Stokes equations', AIAA Paper 85-0165, 1985.

8. B. Koren, 'Multigrid and defect correction for the steady Navier-Stokes equations', J. Comput. Phys., 1990.

9. S. Osher and F. Solomon, 'Upwind difference schemes for hyperbolic systems of conservation laws', Math. Comput., 38, 339-374 (1982).

10. B. van Leer, 'Flux-vector splitting for the Euler equations', Proc. 8th Int. Conf. on Numerical Methods in Fluid Dynamics, Aachen, 1982, Lecture Notes in Physics, Vol. 170, Springer, Berlin, 1982, pp. 507-512.

11. J. L. Thomas and R. W. Walters, 'Upwind relaxation algorithms for the Navier-Stokes equations', AIAA Paper 85$1501,1985$.

12. G. Shaw and P. Wesseling, 'Multigrid solution of the compressible Navier-Stokes equations on a vector computer', Proc. 10th Int. Conf. on Numerical Methods in Fluid Dynamics, Beijing, 1986, Lecture Notes in Physics, Vol. 264, Springer, Berlin, 1986, pp. 566-571.

13. W. Schröder and D. Hänel, 'An unfactored implicit scheme with multigrid acceleration for the solution of the Navier-Stokes equations', Comput. Fluids, 15, 313-336 (1987).

14. B. van Leer, J. L. Thomas, P. L. Roe and R. W. Newsome, 'A comparison of numerical flux formulas for the Euler and Navier-Stokes equations', AIAA Paper 87-1104, 1987.

15. B. van Leer, 'Upwind-difference methods for aerodynamic problems governed by the Euler equations', Proc. 15th AMS-SIAM Summer Seminar on Applied Mathematics, Scripps Institution of Oceanography, 1983, Lectures in Applied Mathematics, Vol. 22, AMS, Providence, RI, 1985, pp. 327-336.

16. S. P. Spekreijse, 'Multigrid solution of monotone second-order discretizations of hyperbolic conservation laws', Math. Comput., 49, 135-155 (1987).

17. P. K. Sweby, 'High resolution schemes using flux limiters for hyperbolic conservation laws', SIAM J. Numer. Anal., 21, 995-1011 (1984).

18. R. J. Hakkinen, I. Greber, L. Trilling and S. S. Abarbanel, 'The interaction of an oblique shock wave with a laminar boundary layer', NASA Memorandum 2-18-59 W, 1959.

19. G. D. van Albada, B. van Leer and W. W. Roberts, "A comparative study of computational methods in cosmic gasdynamics', Astron. Astrophys., 108, 76-84 (1982). 